\title{
Six-Speed Improved Shaft Gearboxes: Optimization of Main Parameters
}

\author{
V.I. Nekrasov ${ }^{1}$, Nikolay Stepanovich Zakharov ${ }^{2}$, A.V. Ziganshina ${ }^{3}$, R.A. Ziganshin ${ }^{4}$ \\ ${ }^{1}$ Federal State Budget Educational Institution of Higher Education «Tyumen Industrial University» (branch \\ office in Surgut), Russia \\ ${ }^{2}$ Tyumen Industrial University, Russia \\ ${ }^{3}$ Federal State Budget Educational Institution of Higher Education «Tyumen Industrial University» (branch \\ office in Surgut), Russia \\ ${ }^{4}$ Federal State Budget Educational Institution of Higher Education «Tyumen Industrial University» (branch \\ office in Surgut), Russia
}

\begin{abstract}
This article describes designs and optimization of main parameters of six-speed improved gear boxes with loose gear arrangement on shafts.
\end{abstract}

Key words: gearbox, improved gearbox, ray path diagram, transmission, gear ratios, range, intervals, gears, shafts, sizes, metal intensity.

\section{INTRODUCTION}

At present there is the steady trend to increase the number of gears in gearbox (GB), which expands operation capabilities of land vehicles.

The main GB parameters are as follows: the number of shafts, $\boldsymbol{n}$ - the number of ratios, $\boldsymbol{U}$ - the gear ratios, $\boldsymbol{U}$ - the gear couples, $\boldsymbol{D}$ - the GB range which is the gear ratios of low to high gears, $\boldsymbol{q}$ - the steps (intervals) which are the gear ratios of neighbor gears, sizes and metal intensity, $\boldsymbol{A}$ - the interaxial distance determining transversal size, $\boldsymbol{g}-$ the number of gears determining the GB longitudinal size.

The coefficient of gear use intensity for conventional GB is moderate: $\boldsymbol{K} \boldsymbol{a}=\boldsymbol{n} / \boldsymbol{g}=6 / 12=0.5$, since each ratio requires for its gear couple.

Designs based on loose arrangement of gears on shafts facilitating gear operation at several ratios significantly increase $\boldsymbol{K} \boldsymbol{a}=6 / 8=0.75$, as well as $\boldsymbol{K} \boldsymbol{a}=8 / 8=1.0$, which reduces GB longitudinal size and metal intensity.

Coefficients of reduction and arrangement efficiency also increase.

The coefficient of reduction $\boldsymbol{K} b=\boldsymbol{n} / \boldsymbol{P}$ equals to the ratio of gear numbers to cumulative reduction. $\boldsymbol{P}$ is the cumulative reduction, it is determined by ray path diagram and equals to the sum of projections of gear couples onto the axis of ratios in logarithmic scale: the sum of intervals (steps) $\boldsymbol{q}$. For the ray path diagram in Fig. 1, the cumulative reduction $\boldsymbol{P}=$ $1.5+0.5+0.5=6, K b=n / P=8 / 6=1.33$.
Multicouple engagement at low ratios decreases gear ratios in gear couples and GB cumulative reduction, and increases $\boldsymbol{K b}$, thus decreasing $\boldsymbol{A}$, i.e. the interaxial distance determining GB transversal size.

The coefficient of arrangement efficiency equals to the sum of aforementioned coefficients $\boldsymbol{K c}=\boldsymbol{K a}+\boldsymbol{K} \mathbf{b}=1.0+1.33=$ 2.33. The higher is the $\boldsymbol{K c}$, the better is the GB arrangement.

\section{METHODS}

\subsection{Eight-speed IGBs}

Six-speed improved gearboxes (IGBs) are developed on the basis of eight-speed IGBs. Figure 1 illustrates kinematic flowcharts and ray path diagrams of eight-speed IGBs.

Four banks of gears (I, II, III, IV) arranged loosely on shafts and three shift clutches (A, B, C) in 8R4 IGB provide eight forward gears and four reverse gears by the bank of gears $R$ with idle gear in separate axle.

If the IGB range is $\boldsymbol{D}=6.0 ; \lg 6=0.778$. Herewith, $\lg \mathrm{q}=$ $0.778 / 7=0.111$ and the interval $\mathrm{q}=1.292$. These indices would provide wide scope of operation conditions and convenience of gear shifting. Gear ratios of gear couples of three-shaft IGB are: $\lg \boldsymbol{U}_{\mathrm{I}}=1.5 \mathrm{lg} \mathrm{q}=1.5 \times 0.111=0.167 ; \boldsymbol{U}_{\mathrm{I}}$ $=1.467 ; \lg \boldsymbol{U}_{\mathrm{II}}=\lg \boldsymbol{U}_{\mathrm{III}}=0.5 \times 0.111=0.056 ; \boldsymbol{U}_{\mathrm{II}}=\boldsymbol{U}_{\mathrm{III}}=$ $1.136 ; \lg \boldsymbol{U}_{\mathrm{IV}}=3.5 \lg \mathrm{q}=3.5 \times 0.111=0.389 ; \boldsymbol{U}_{\mathrm{IV}}=2.449$.

The first ratio is provided by four gear couples: the ray I from the point $\mathbf{7 g}$ downward to the right, the ray II upward to the right to the point $\mathbf{5 g}$, the ray III downwards, then the ray IV upward to the point $1 \mathrm{~g}$. The gear ratio is $\boldsymbol{U}_{1} \mathrm{~g}=\boldsymbol{U}_{\mathrm{I}} \boldsymbol{U}_{\mathrm{II}} \boldsymbol{U}_{\mathrm{III}}$ $\boldsymbol{U}_{\mathrm{IV}}=1.467 \times 1.136 \times 1.136 \times 2.449=4.64$ or $\lg \boldsymbol{U}_{1} \mathrm{~g}=\lg \boldsymbol{U}_{\mathrm{I}}+$ $\lg \boldsymbol{U}_{\mathrm{II}}+\lg \boldsymbol{U}_{\mathrm{III}}+\lg \boldsymbol{U}_{\mathrm{IV}}=0.167+0.056+0.056+0.389=0.668$; $\boldsymbol{U}_{1} \mathrm{~g}=4.64$.

The accelerating eighth gear is provided by two gear couples: the ray II from the point $\mathbf{7 g}$ downward to the left, the ray III upward to the left to the point $\mathbf{8 g}$. The gear ratio is $\boldsymbol{U}_{8} \mathrm{~g}=$ $1 /\left(\boldsymbol{U}_{\mathrm{II}} \boldsymbol{U}_{\mathrm{III}}\right)=1 /(1.136 \times 1.136)=0.775$ or $\lg \boldsymbol{U}_{8} \mathrm{~g}=\lg \boldsymbol{U}_{\mathrm{II}}+\lg$ $\boldsymbol{U}_{\mathrm{III}}=0.056+0.056=0.111 ; \boldsymbol{U}_{8} \mathrm{~g}=0.775$.

IGB range is: $\boldsymbol{D}=4.64 / 0.775=6.0$. It is possible to expand the interval and range of $8 \mathrm{R} 4 \mathrm{IGB}$. 


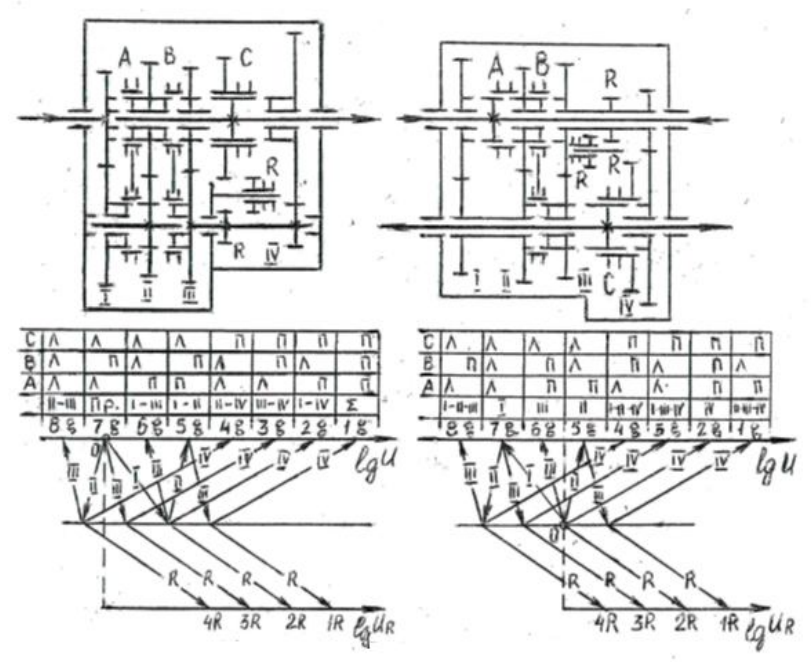

Figure 1: Kinematic flowcharts and ray path diagrams with tables of clutch positions of eight-speed 8R4 IGB: left side three-shaft coaxial, right side - two-shaft noncoaxial.

The eight-speed IGB has a disadvantage: end engagement of reverse gears $\mathrm{R}$. The reverse gears are engaged by the idle gear $\mathrm{R}$ installed on separate axle upon its movement up to engagement with driving reverse gear fixed on intermediate gear, and with driven wheel fixed on the shift clutch $\mathrm{C}$ in neutral position. The end engagement leads to increased wear of tooth ends and noise. The wear products of tooth ends lead to increased wear of all gear teeth, decrease operation lifetime of transmission oil and of overall IGB. The end engagement is not used in modern GB.

\subsection{Six-speed IGBs}

6R2 IGBs were developed on the basis of 8R4 IGBs. In 6R2 IGB, the end engagement is not applied: all gears are constantly engaged, they are activated by toothed clutches. Figure 2 illustrates kinematic flowcharts and ray path diagrams of six-speed IGBs with tables of shift clutch positions.

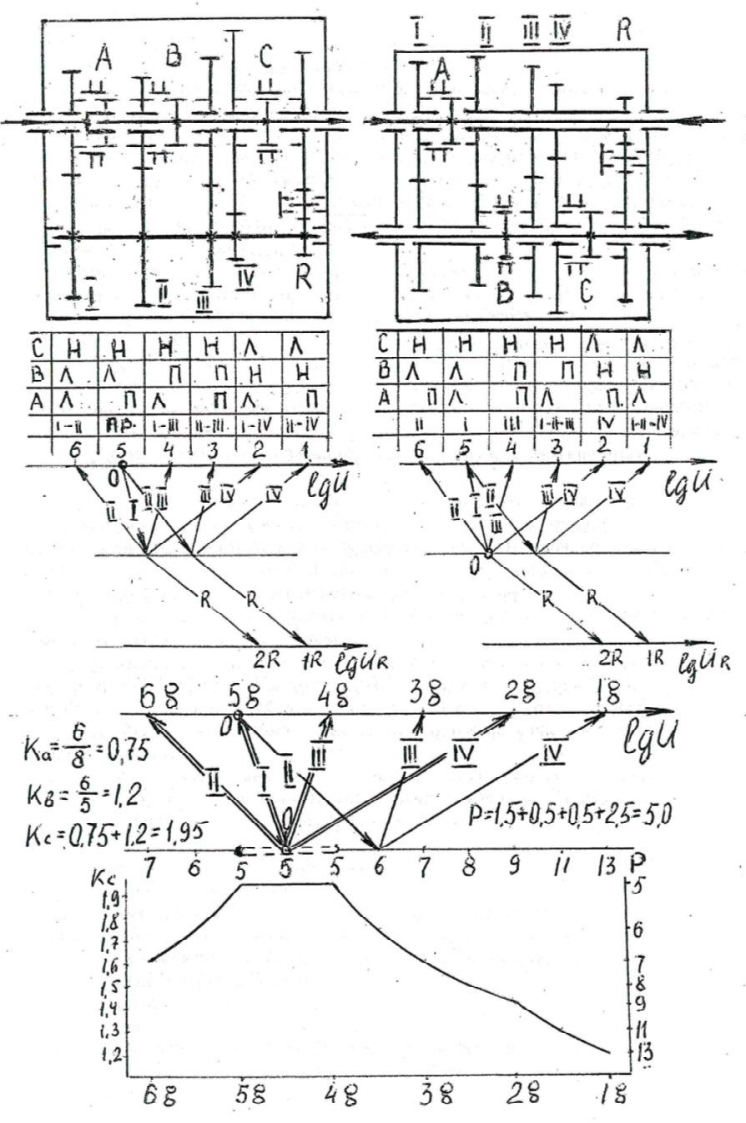

Figure 2: Kinematic flowcharts and ray path diagrams with clutch positions of 6R2 IGB: left side - three-shaft coaxial, right side - two-shaft noncoaxial; bottom - generalized ray path diagram and its evaluations.

Under the ray path diagram, the cumulative reduction $P$ and the coefficient of arrangement efficiency $K c$ are shown as a function of point 0 of outlet of determinant rays on the lower horizontal line. Predictions of these variables are summarized in Table 1.

Table 1. Rays, cumulative reduction $P$, and coefficient of arrangement efficiency $K c$ as a function of outlet of determinant rays

\begin{tabular}{|l|l|l|l|l|l|l|l|}
\hline $\begin{array}{l}\text { Point } \\
0\end{array}$ & I & II & III & IV & $P$ & $\boldsymbol{K b}$ & $\boldsymbol{K c}$ \\
\hline 1.0 & 4.0 & 5.0 & 3.0 & 1.0 & 13 & 0.46 & 1.21 \\
\hline 1.5 & 3.5 & 4.5 & 2.5 & 0.5 & 11 & 0.5 & 1.3 \\
\hline 2.0 & 3.0 & 4.0 & 2.0 & $\mathbf{0}$ & 9 & 0.67 & 1.42 \\
\hline 2.5 & 2.5 & 3.5 & 1.5 & 0.5 & 8 & 0.75 & 1.5 \\
\hline 3.0 & 2.0 & 3.0 & 1.0 & 1.0 & 7 & 0.86 & 1.61 \\
\hline 3.5 & 1.5 & 2.5 & 0.5 & 1.5 & 6 & 1.0 & 1.75 \\
\hline 4.0 & 1.0 & 2.0 & $\mathbf{0}$ & 2.0 & $\mathbf{5}$ & $\mathbf{1 . 2}$ & $\mathbf{1 . 9 5}$ \\
\hline 4.5 & 0.5 & 1.5 & 0.5 & 2.5 & $\mathbf{5}$ & $\mathbf{1 . 2}$ & $\mathbf{1 . 9 5}$ \\
\hline 5.0 & $\mathbf{0}$ & 1.0 & 1.0 & 3.0 & $\mathbf{5}$ & $\mathbf{1 . 2}$ & $\mathbf{1 . 9 5}$ \\
\hline 5.5 & 0.5 & 0.5 & 1.5 & 3.5 & 6 & 1.0 & 1.75 \\
\hline 6.0 & 1.0 & $\mathbf{0}$ & 2.0 & 4.0 & 6 & 0.86 & 1.61 \\
\hline
\end{tabular}

The optimum variant with $K c=1.95$ is obtained for the points between $\mathbf{5 g}$ and $\mathbf{4 g}$. Position of ray outlet point significantly effects the cumulative reduction $P$, it is shown 
under the lower horizontal line together with the coefficients of reduction and arrangement efficiency.

For instance, if for the optimum variant upon the gear $\mathbf{5 g}$ with cumulative reduction $P=0+1+1+3=5, \mathrm{~Kb}=1.2$ and $\boldsymbol{K} \mathrm{c}=1.95$ this point is displaced by one step to the left, then we obtain the gear $\mathbf{6 g}$ with $P=1.0+0+2+4=7, K b=0.86$ and $=1.61$. Upon displacement from the point at the gear $\mathbf{4 g}$ with cumulative reduction $P=1+2+0+2=5, K \mathrm{~b}=1.2$ and $\boldsymbol{K c}=1.95$ by one step to the right, we obtain the gear $\mathbf{3 g}$ with $P=2+3+1+1=7, K \mathrm{~b}=0.86$ and $K \mathrm{c}=1.61$; by two steps to the right - the gear $2 \mathrm{~g}$ with $P=3+4+2+0=9, K \mathrm{~b}=0.67$ and $K \mathrm{c}=1.42$.

When transferring from $8 \mathrm{R} 4$ IGB to $6 \mathrm{R} 2 \mathrm{IGB}$, it is impossible to obtain two forward gears with the rays III, IV (the gear $\mathbf{3 g}$ ) and the rays I, II, III, IV (the gear 1g) for threeshaft IGB and with the rays I, III, IV (the gear $\mathbf{3 g}$ ) and the rays II, III, IV (the gear 1g) for two-shaft IGB. Consecutive engagement of the gear couples III and IV is excluded from operation. If additional clutch between these gear couples is added, then we obtain 8R4 IGB.

Four banks of gears (I, II, III, IV) loosely installed on shafts and three shift clutches $(\mathrm{A}, \mathrm{B}, \mathrm{C})$ in $6 \mathrm{R} 2$ IGB provide six forward gears and two reverse gears by the gears $\mathrm{R}$. The coefficient of gear use is $\boldsymbol{K a}=\boldsymbol{n} / \boldsymbol{g}=6 / 8=0.75$.

Comparison of the ray path diagrams of three- and two-shaft IGBs highlights their general pattern. The only difference is in the outlet point of the ray I: from the left out of the point 0 , corresponding to the fifth forward gear, downward to the right to middle horizontal line; from the right, out of the lower horizontal line out of the point 0 upward to the right to the point of the fifth gear. Both IGBs can be presented by one generalized ray path diagram shown at the bottom of Fig. 2. Two lines indicate the determinant rays originating from common point at lower horizontal line: the ray I is directed to the point $\mathbf{5 g}$ with two arrows, the ray II in accelerating mode is directed upward to the left to the gear $\mathbf{6 g}$, the ray III is directed upward to the gear $\mathbf{4 g}$, then the ray IV is directed to the gear $\mathbf{2 g}$.

Under the lower horizontal line, the cumulative reduction is shown for various outlet positions of determinant rays, the same values are shown on the right vertical scale. The minimum cumulative reduction corresponds to the points at the gears $\mathbf{5 g}$ and $\mathbf{4 g}$.

For Fig. 2 the cumulative reduction is $P=1.5+0.5+0.5+2.5=$ 5.0. The coefficient of reduction is $\boldsymbol{K b}=\boldsymbol{n} / \boldsymbol{P}=6 / 5=1.2$. The coefficient of arrangement efficiency is $K c=K a+K b=$ $0.75+1.2=1.95$. This coefficient is shown on the left vertical scale.Similar generalized ray path diagrams can be plotted for all 48 arrangement variants of 8R4 IGB and 6R2 IGB.
If the IGB range is $\boldsymbol{D}=6.0 ; \lg 6=0.778$. Herewith, $\lg \mathrm{q}=$ $0.778 / 5=0.1556$; and the interval is $q=1.431$. These indices would provide wide scope of operation conditions and convenience of gear shifting. Gear ratios of gear couples of three-shaft IGB are: $\lg \boldsymbol{U}_{\mathrm{I}}=\lg \boldsymbol{U}_{\mathrm{III}}=0.5 \times 0.1556=0.0778 ; \boldsymbol{U}_{\mathrm{I}}$ $=\boldsymbol{U}_{\mathrm{III}}=1.196 ; \lg \boldsymbol{U}_{\mathrm{II}}=1.5 \lg \mathrm{q}=1.5 \times 0.1556=0.2334 ; \boldsymbol{U}_{\mathrm{II}}=$ $1.71 ; \lg \boldsymbol{U}_{\mathrm{IV}}=2.5 \lg \mathrm{q}=2.5 \times 0.1556=0.389 ; \boldsymbol{U}_{\mathrm{IV}}=2.449$.

For the three-shaft IGB (Fig. 2, at the left), the first gear is provided by two gear couples: the ray II from the point $\mathbf{5 g}$ downward to the right, then the ray IV upward to the point 1g. The gear ratio is $\boldsymbol{U}_{1} \mathrm{~g}=\boldsymbol{U}_{\mathrm{II}} \boldsymbol{U}_{\mathrm{IV}}=1.71 \times 2.449=4.19$ or $\mathrm{lg}$ $\boldsymbol{U}_{1} \mathrm{~g}=\lg \boldsymbol{U}_{\text {II }}+\lg \boldsymbol{U}_{\text {IV }}=0.2334+0.389=0.6224 ; \boldsymbol{U}_{1} \mathrm{~g}=4.19$.

The accelerating sixth gear is provided by two gear couples: the ray I from the point $\mathbf{5 g}$ downward to the right, the ray II upward to the left to the point $6 \mathrm{~g}$. The gear ratio is $\boldsymbol{U}_{6} \mathrm{~g}=$ $\boldsymbol{U}_{\mathrm{I}} / \boldsymbol{U}_{\mathrm{II}}=1.196 / 1.71=0.699$ or $\lg \boldsymbol{U}_{6} \mathrm{~g}=\lg \boldsymbol{U}_{\mathrm{I}}+\lg \boldsymbol{U}_{\mathrm{II}}=$ $0.0778-0.2334=-0.1556 ; \boldsymbol{U}_{6} \mathrm{~g}=0.699$. IGB range is $\boldsymbol{D}=$ $4.19 / 0.699=6.0$.

For the two-shaft IGB (Fig. 2, at the right), the first gear is provided by three gear couples: the ray I from the point 0 on the middle horizontal line upward to the left, the ray II from the point $\mathbf{5 g}$ downward to the right, then the ray IV upward to the point $1 \mathrm{~g}$. The gear ratio is $\boldsymbol{U}_{1} \mathrm{~g}=\boldsymbol{U}_{\mathrm{I}} \boldsymbol{U}_{\mathrm{II}} \boldsymbol{U}_{\mathrm{IV}}=$ $(1 / 1.196) \times 1.71 \times 2.449=3.5$ or $\lg \boldsymbol{U}_{1} \mathrm{~g}=\lg \boldsymbol{U}_{\mathrm{I}}+\lg \boldsymbol{U}_{\mathrm{II}}+\lg$ $\boldsymbol{U}_{\mathrm{IV}}=-0.0778+0.2334+0.389=0.5446 ; \boldsymbol{U}_{1} \mathrm{~g}=3.5$.

The accelerating sixth gear is provided by one gear couple: the ray II upward to the left to the point $\mathbf{6 g}$. The gear ratio is $\boldsymbol{U}_{6} \mathrm{~g}=1 / \boldsymbol{U}_{\mathrm{II}}=1.0 / 1.71=0.585$ or $\lg \boldsymbol{U}_{6} \mathrm{~g}=\lg \boldsymbol{U}_{\mathrm{II}}=-0.2334$; $\boldsymbol{U}_{6} \mathrm{~g}=0.585$. IGB range is $\boldsymbol{D}=3.5 / 0.585=6.0$. It is possible to expand the interval and range of 6R2 IGB.

\section{RESULTS AND DISCUSSION}

\subsection{Optimization of main parameters of six-speed IGBs}

Generalized ray path diagrams and their notations for twoand three-shaft 6R2 IGBs are summarized in Table 2.

Table 2 is based on the table for 8R4 IGB. The left column corresponds to the number of direct gear of three-shaft IGB. The upper line shows operation variants of shift clutches. Six operation variants of shift clutches are possible: $\mathrm{ABC}, \mathrm{BAC}$, $\mathrm{ACB}, \mathrm{CAB}, \mathrm{BCA}$ and $\mathrm{CBA}$. In general, it is possible to obtain $8 \times 6=48$ arrangement variants of 8R4 IGB. Each of the 48 arrangement variants has its optimum arrangement coefficient $K \mathrm{C}$.

The above considered variant is shown in the seventh line and the first column: 7ABC 1-2-4. The forward gear was $7 \mathrm{~g}$, now it is 5g. The obtained variant is 5ABC 1-2-4. 1,95 (5-4) 5-6-4-2.

$\mathbf{A B C}$ is the operation order of shift clutches. The first letter (A) denotes the clutch engaged upon each shift, the second letter (B) denotes the clutch engaged after two consecutive shifts, the third letter (C) denotes the clutch engaged after 
four consecutive shifts (see tables of ray path diagrams, Fig. 2).

The numbers 1-2-4 denote intervals between the rays: one interval between the rays I and II (5g - 6g); two intervals between the rays II and III $(6 \mathrm{~g}-4 \mathrm{~g})$; four intervals between the rays III and IV for 8R4 IGB; two intervals remain for 6R2 IGB $(4 \mathrm{~g}-2 \mathrm{~g})$.

1.95 is the maximum coefficient of arrangement efficiency $\mathrm{Kc}=1.95$.

(5 - 4) are the outlet points of determinant rays from the lower diagonal with maximum $\mathrm{Kc}=1.95$.
5-6-4-2 are the gears to which the determinant rays are directed. Four determinant rays originate from the point 0 on the lower horizontal line: I to the point $5 \mathrm{~g}$, it is denoted by double line with two arrows; II to the point $6 \mathrm{~g}$; III to the point $4 \mathrm{~g}$; IV to the point $2 \mathrm{~g}$, denoted by double lines with arrows.

The data in Table 2 make it possible to substantiate selection of optimum variants of 6R2 IGB.

Some ray path diagrams coincide, for instance: $1 \mathrm{BCA}$ and 1 $\mathrm{CBA}, 2 \mathrm{BCA}$ and $2 \mathrm{CAB}$. A portion of ray path diagrams having common pattern are characterized by different number of rays, for instance, $3 \mathrm{ABC}$ and $5 \mathrm{ABC}$ are the rays III and IV.

Table 2: Ray path diagrams and conventional notations of arrangement variants of 6R2 IGB based on 8R4 IGB

\begin{tabular}{|c|c|c|c|}
\hline N & $A B C \cdot 1-2 \cdot 4$ & $B A C \quad 2.1 .4$ & $A C B \quad 1-4.2$ \\
\hline 1 & $\frac{6^{5}{ }^{3}{ }^{3}}{1,61(4-2)^{1}(-2-4-6}$ & $\int_{1,61(4-3)}^{5} \int_{1-3-4-6^{*}}^{3}$ & $1,61(4-2) 1-2-4-6$ \\
\hline 2 & 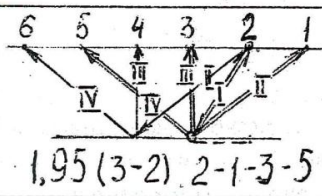 & 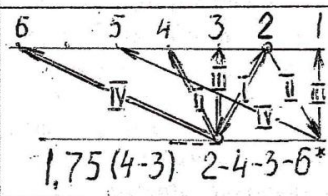 & $1,61(4-2) 1-2-4-6$ \\
\hline 3 & $\frac{5432}{1,95(4-3) 3-4-2-6}$ & $\frac{3^{5} \int^{3}}{1,95(3-2)}$ & $1,61(4-2) \frac{1}{1-2-6-4}$ \\
\hline 4 & 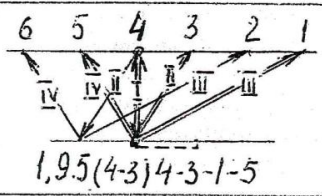 & $\frac{543.2}{1,75(4-2)}$ & $1,95(4-3) 2-1-5-3$ \\
\hline 5 & $\frac{6 e^{4} 2^{3}}{1,95(4-3) 3-4-6-2}$ & $\begin{array}{l}642 \\
1.75(5-3) \\
3-5-6-2^{*}\end{array}$ & $\begin{array}{c}63^{5} 3^{3} 2 \\
\qquad, 95(5-4) \\
5-6-2-4\end{array}$ \\
\hline 6 & $\frac{64}{1.95(4-3) \quad 4-3-5 \cdot 1}$ & 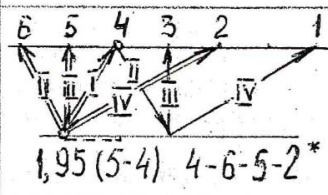 & $\frac{6.52 .32}{1,01(5-3) 6-5-1-3}$ \\
\hline 7 & 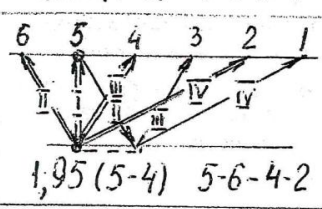 & $\underbrace{5}_{1,75(4-3)\left(5-3-4-1^{*}\right.}$ & $\frac{5432}{1,95(5-4) 5-6-4-2}$ \\
\hline 8 & 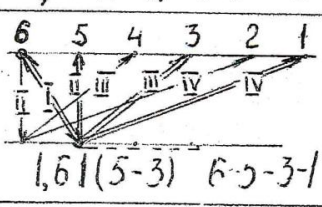 & 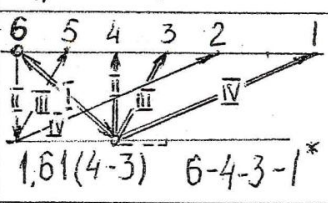 & 年 \\
\hline
\end{tabular}




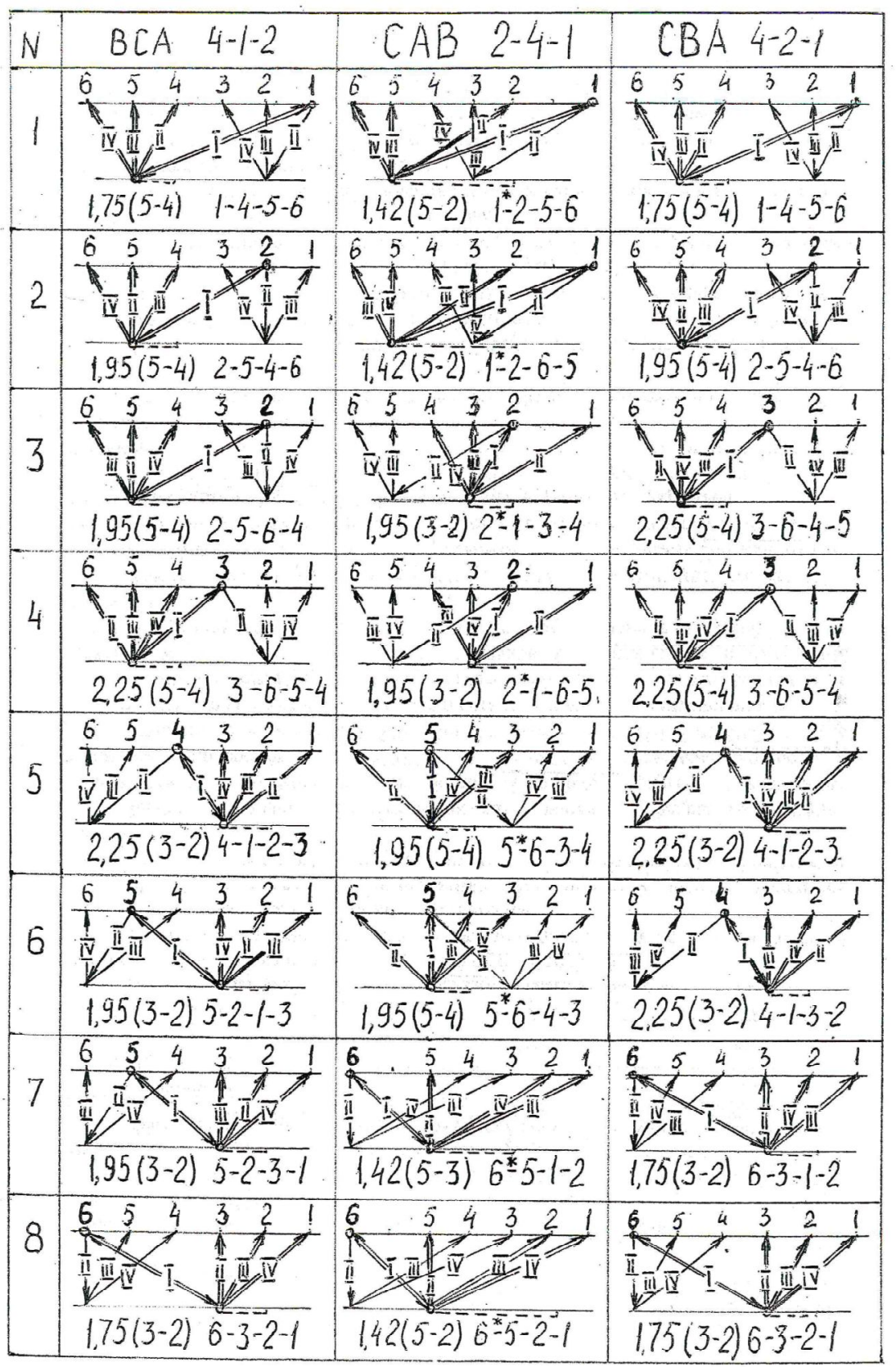

The variants BCA 4 and 5; CBA 3-6 are characterized by the maximum coefficient of arrangement efficiency $K \mathrm{c}=2.2$, however, they do not meet other requirements. High efficiency at high gears is required: two-shaft IGBs should have one ray-one toothed gear; for three-shaft IGBs: two consecutive rays-two toothed gears. High gear should be positioned in the left part of the ray path diagram: at the level of $6 \mathbf{g}-\mathbf{5 g}$. Such gears are located at the bottom of Table 2. In addition, it is required to aim at the variant with minimum value of the ray IV which determines the IGB transversal size and metal intensity. [1-3]
The optimum variant is positioned at the level of $\mathbf{7} \mathbf{A B C}$ and 7 ACB with 5ABC 1-2-4. 1,95 (5-4) 5-6-4-2 arrangement considered above. The variant $\mathbf{5}$ ACB is close to the above considered variants, it differs only by the rays III and IV.

For three-shaft IGBs with high forward gear $\mathbf{6 g}$, the reasonable variants are $8 \mathrm{ABC}$ and $8 \mathrm{ACB}$ with $6 \mathrm{ABC}$ 1-2-4. 1,61 (5-3) 6-5-3-1 arrangement.

For freight vehicles, the variants of interest are those with increased interval between low gears $\mathbf{1 g}$ and $\mathbf{2 g}$. For threeshaft IGB, this is 7 BAC with 5BAC 2-1-4. 1,75 (4-3) 5-3-41* arrangement, for two-shaft IGB: 6 BAC with 4 ABC 2-14. 1,95 (5-4) 4-6-5-2* arrangement. $[4,5]$. 


\subsection{Technical and economical evaluation of main parameters of IGBs}

The method of loose arrangement of gears on shafts makes it possible to develop IGB of minimum sizes and weight. Operation of banks of gears at several ratios makes it possible to obtain the same number of ratios using lower number of gears as well as to decrease gear ratios of gear couples, mostly gear couple at IGB outlet, which reduces IGB metal intensity due to decreased longitudinal and transversal sizes.

If with the aim of comparison we consider five-speed conventional GB of YaMZ-236N as prototype, then we have: intermediate shaft drive $U_{\mathrm{sd}}=38 / 28=1.357$; the drive from the intermediate shaft to the first gear $U_{\mathrm{I}}=62 / 16=3.875$; the gear ratio of the first gear $\boldsymbol{U}_{1} \mathrm{~g}=U_{\text {sd }} U_{\mathrm{I}}=1.357 \times 3.875=$ 5.26. The drive from intermediate shaft to the fifth accelerating gear is $U_{5}=23 / 47=0.489$; the gear ratio of the fifth gear is $\boldsymbol{U}_{5} \mathrm{~g}=U_{\mathrm{sd}} U_{5}=1.357 \times 0.489=0.664$. The range is $D=3.875 / 0.664=7.92$. The average interval is $q=D^{1 /(\mathrm{n}-1)}$ $=7.92^{1 / 4}=7.92^{0.25}=1.68 .[6,7]$.

For 6R2 IGB at the same range $D=7.92$, we have the average interval $q=D^{1 /(\mathrm{n}-1)}=7.92^{1 / 5}=7.92^{0.2}=1.51$ which improves conditions of gear sifts. The gear ratio of the IV gear couple is $\boldsymbol{U}_{\mathrm{IV}}=q^{2.5}=1.51^{2.5}=2.8$ which is significantly lower than that of the prototype: $U_{\mathrm{I}}=3.875$.

6R2 IGB is characterized by better performances in comparison with regular designs: increased intensity of gear use, decreased cumulative reduction, which facilitates significant reduction of GB sizes and metal intensity.

Therefore, the optimum variants of six-speed 6R2 IGB are 7 $\mathrm{ABC}$ and 7 ACB with 5ABC 1-2-4. 1,95 (5-4) 5-6-4-2 arrangement meeting the requirements of minimum weigh, high efficiency, and convenience of gear shifting.

\section{CONCLUSION}

The proposed approach can be applied for selection of optimum variants of 6-, 12-, 24-speed and more powerful IGBs, for development of compact assemblies with low metal intensity protected by RF patents.

\section{CONFLICT OF INTEREST}

The authors confirm that the submitted data do not contain conflict of interest.

\section{REFERENCES}

[1] A.I. Grishkevich. Avtomobili: Konstruktsiya, konstruirovanie $i$ raschet. Transmissiya [Automobiles: Designs, engineering, and development. Transmission]. Moscow, Vysh. shk., 1985.

[2] V.I. Nekrasov. Mnogostupenchataya transmissiya. Konstruktsiya, konstruirovanie $i$ raschet [Multistage transmission. Design, engineering and development]: Guidebook. Kurgan, Kurgan State University, 2001.

[3] V.I. Nekrasov. Optimization of main parameters of gearboxes with three gear trains. ARPN Journal of Engineering and Applied Sciences, Vol. 12(11), pp. 35263531, June 2017.

[4] R.A. Ziganshin. Six-speed power gearboxes. International Journal of Mechanical Engi-neering and Technology, Vol. 8(10), pp. 401-409, October 2017.

[5] R.A. Ziganshin. Assessment of the transmission's impact on the operational properties of land vehicles. International Journal of Engineering and Technology (UAE), 2018

[6] R.A. Ziganshin. Methods of optimization of vehicle parameters. International Journal of Mechanical Engineering and Technology, 2018

[7] R.A. Ziganshin. Noncoaxial two-shaft six-speed gearboxes. International Journal of Emerging Trends in Engineering Research, 2019. 